\title{
CLONADO, EXPRESIÓN, CARACTERIZACIÓN MOLECULAR Y FISICOQUÍMICA DE ENZIMAS INVOLUCRADAS EN LA ETAPA DE DESNITRIFICACIÓN DEL CICLO DEL NITRÓGENO
}

\author{
Cristaldi, Julio César \\ Director: Dr. Carlos D. Brondino \\ Co-Directora: Dra. María Gabriela Rivas \\ Lugar de realización: Departamento de Física, Facultad de Bioquímica y Ciencias Biológicas, Universidad Nacional del Litoral (FBCB-UNL). \\ Fecha de la defensa: 27 de marzo de 2019
}

\section{RESUMEN}

En este trabajo se estudiaron distintos aspectos a nivel molecular del mecanismo catalítico de la nitrito reductasa de cobre (NirK), una enzima que cataliza la reducción de nitrito $\left(\mathrm{NO}_{2}{ }^{-}\right)$a óxido nítrico (NO) en la desnitrificación. Estas enzimas se clasifican de acuerdo a sus propiedades espectroscópicas en verdes y azules. El trabajo se desarrolló a partir del estudio de dos Nirk producidas de manera recombinante, una verde obtenida del microorganismo Sinorhizobium meliloti 2011 (SmNirK), y otra azul obtenida de Bradyrhizobium japonicum USDA 110 (BjNirK). Los dos microorganismos son bacterias desnitrificantes de importancia económica para la región por su uso en la formulación de bioinoculantes. Ambas Nirk presentan una estructura homotrimérica con dos sitios de cobre por monómero denominados T1Cu y $\mathrm{T} 2 \mathrm{Cu}$, en línea con la mayoría de las NirK caracterizadas. El centro T1Cu, el cual le confiere el color característico a la enzima, es un centro de transferencia electrónica (TE). El T2Cu es el sitio activo en donde ocurre la unión y reducción del sustrato. Las Nirk requieren de un dador electrónico fisiológico para llevar a cabo la catálisis, los cuales también fueron producidos de manera recombinante y caracterizados. El dador electrónico fisiológico de la SmNirK es una proteína de cobre mononuclear denominada pseudoazurina ( $S m P a z)$, mientras que para BjNirK es un citocromo $c$ (BjCitc $\left.{ }_{550}\right)$.

El mecanismo catalítico de las Nirk puede ser dividido en líneas generales en tres procesos. El primer proceso de TE resulta de la interacción de la Nirk con el dador electrónico fisiológico. Esto implica la transferencia de un electrón desde el dador electrónico reducido al T1Cu de la Nirk (TE interproteína). El segundo proceso de TE ocurre desde el T1Cu reducido al sitio activo T2Cu (TE intraproteína), lo que permite la reducción del sustrato $\left(\mathrm{NO}_{2}^{-}\right)$unido al sitio y liberación del producto (NO) (tercer proceso). El proceso de TE intraproteína ocurre a través del puente estructural Cis-His que conecta los dos sitios de $\mathrm{Cu}$ en las Nirk. En este puente coexisten dos vías estructurales que potencialmente pueden actuar como camino de TE. Una de estas vías involucra el camino covalente Cis-His, mientras que la segunda vía involucraría un camino mixto formado por enlaces covalentes y el puente de hidrógeno establecido entre el $\mathrm{O}$ del carbonilo de la Cis y el $\mathrm{N}_{\delta 1}$ del imidazol de la $\mathrm{His}\left(\mathrm{N}_{\delta 1} \mathrm{H} \ldots \mathrm{O}=\mathrm{C}\right)$. Sobre la base de estudios computacionales se ha propuesto que las NirK azules utilizarían la primer vía mientas que las verdes utilizarían la segunda. 
La determinación de los potenciales formales de reducción $\left(\mathrm{E}^{\mathrm{O}^{\prime}}\right)$ de los centros de cobre de la forma aspurified de SmNirK mediante titulaciones potenciométricas permitió concluir que, en línea con la mayoría de las NirK, $\mathrm{E}^{\mathrm{O}^{\prime}} \mathrm{T1Cu}_{\mathrm{C}}>\mathrm{E}^{\mathrm{O}^{\prime}} \mathrm{T2 \textrm {Cu }}$. Estos valores definen un proceso de $\mathrm{TE} \mathrm{T} 1 \mathrm{Cu} \rightarrow \mathrm{T} 2 \mathrm{Cu}$ termodinámicamente desfavorable en la enzima. Los resultados obtenidos a partir de ensayos de EPR sugieren que los valores relativos de los $\mathrm{E}^{0^{\prime}}$ se invierten como consecuencia de la interacción SmPaz/SmNirK en presencia de sustrato favoreciendo el proceso de TE.

La TE intraproteína en esta enzima también fue estudiado cambiando los aminoácidos del puente CisHis mediante mutagénesis sitio dirigida. Las variantes obtenidas resultaron inactivas frente a SmPaz. Modelos estructurales obtenidos mediante estudios computacionales del tipo QM/MM, junto con los resultados experimentales, sugirieren que la falta de actividad se debería a una interrupción de la TE intraproteína, posiblemente por la ausencia del puente de hidrógeno $\mathrm{N}_{\delta 1} \mathrm{H} \ldots \mathrm{O}=\mathrm{C}$.

Los resultados obtenidos para las variantes de SmNirK derivaron en el estudio de la relevancia de la estructura $\mathrm{N}_{\delta 1} \mathrm{H} \ldots . \mathrm{O}=\mathrm{C}$ en la TE intraproteína de las NirK verdes y azules y en la dependencia de la actividad catalítica de las Nirk con el pH del medio. Esto fue realizado mediante estudios dependientes del $\mathrm{pH}$ en las dos Nirk en los cuales se monitorearon los estados de oxidación de los dos centros mediante espectroscopía UV-vis y EPR luego de varios ciclos redox de la enzima en condiciones catalíticas. Los resultados más importantes de estos estudios mostraron que a pH 10, condición en la cual las NirK se vuelven inactivas, el T2Cu mantiene la capacidad de unir y reducir el sustrato aunque a una tasa no detectable por los ensayos cinéticos utilizados. Esto se atribuye a que el alto $\mathrm{pH}$ produce el desacople entre el proceso de TE intraproteina y del proceso de unión nitrito-T2Cu, lo cual se originaría por la ruptura del puente $\mathrm{N}_{\delta 1} \mathrm{H} \ldots \mathrm{O}=\mathrm{C}$. Otro de los resultados relevantes es que en las dos Nirk el puente $\mathrm{N}_{\delta 1} \mathrm{H} \ldots \mathrm{O}=\mathrm{C}$ se comportaría como el único camino de TE, lo que implicaría que la estructura electrónicas del T1Cu no determina el camino de TE.

\section{ABSTRACT}

\section{Cloning, expression and molecular and physicochemical characterization of enzymes involved in the denitrification process of the nitrogen cycle}

Copper nitrite reductase (NirK) is an enzyme that catalyzes the reduction of $\mathrm{NO}_{2}{ }^{-}$to $\mathrm{NO}$ in the denitrification process. These enzymes are classified in green and blue according to their spectroscopic properties. They present two copper sites called T1Cu and T2Cu which are the electron transfer (ET) site and the active site, respectively. The proposed reaction mechanism for Nirs implies a two-proton coupled redox reaction in which nitrite, after binding $\mathrm{T} 2 \mathrm{Cu}$, is converted to NO by one electron delivered to $\mathrm{T} 1 \mathrm{Cu}$ by an external physiological electron donor through the Cis-His bridge. The Cys-His bridge is an electron 
transfer pathway having two potential ET subpathways. In this work a green NirK from Sinorhizobium meliloti 2011 (SmNirK) and a blue enzyme from Bradyrhizobium japonicum USDA 110 (BjNirK) were studied. The main objective was to study the catalytic mechanism of both enzymes characterizing the molecular, spectroscopic and electrochemical properties of both enzymes and their physiological electron donors. EPR studies show that the reduction potentials of the metal centers of SmNirk are modulated by the interaction with the physiological electron donor to favor intraprotein ET. The intraprotein ET process was studied by site-directed mutagenesis changing the amino acids of the Cis-His bridge of SmNirK, in silico structural studies and pH-dependent studies in both NirK. Regardless of the color of the enzymes, intraprotein ET occurs through the same structural subpathway. 